\title{
Density-transition based electron injector for laser driven wakefield accelerators
}

\author{
K. Schmid, ${ }^{1, *}$ A. Buck, ${ }^{1}$ C. M. S. Sears, ${ }^{1}$ J. M. Mikhailova, ${ }^{1,2}$ R. Tautz, ${ }^{1,3}$ D. Herrmann, ${ }^{1,4}$ M. Geissler, ${ }^{5}$ \\ F. Krausz, ${ }^{1,6}$ and L. Veisz ${ }^{1, \dagger}$ \\ ${ }^{1}$ Max-Planck-Institut für Quantenoptik, Hans-Kopfermann-Strasse 1, 85748 Garching, Germany \\ ${ }^{2}$ A.M. Prokhorov General Physics Institute, Russian Academy of Science, Moscow, Russia \\ ${ }^{3}$ LS für Photonik und Optoelektronik, LMU München, Amalienstrasse 54, 80799 München, Germany \\ ${ }^{4}$ LS für BioMolekulare Optik, LMU München, Oettingenstrasse 67, 80538 München, Germany \\ ${ }^{5}$ Queen's University Belfast, Belfast BT7 1NN, United Kingdom \\ ${ }^{6}$ Ludwig-Maximilians-Universität München, Am Coulombwall 1, 85748 Garching, Germany
}

(Received 5 July 2010; published 7 September 2010)

\begin{abstract}
We demonstrate a laser wakefield accelerator with a novel electron injection scheme resulting in enhanced stability, reproducibility, and ease of use. In order to inject electrons into the accelerating phase of the plasma wave, a sharp downward density transition is employed. Prior to ionization by the laser pulse this transition is formed by a shock front induced by a knife edge inserted into a supersonic gas jet. With laser pulses of $8 \mathrm{fs}$ duration and with only $65 \mathrm{~mJ}$ energy on target, the accelerator produces a monoenergetic electron beam with tunable energy between 15 and $25 \mathrm{MeV}$ and on average $3.3 \mathrm{pC}$ charge per electron bunch. The shock-front injector is a simple and powerful new tool to enhance the reproducibility of laser-driven electron accelerators, is easily adapted to different laser parameters, and should therefore allow scaling to the energy range of several hundred $\mathrm{MeV}$.
\end{abstract}

DOI: 10.1103/PhysRevSTAB.13.091301

PACS numbers: 41.75.Ht, 42.65.Jx, 42.65.Re, 42.65.Yj

Laser wakefield accelerators [1] are intensely investigated due to their ability to produce longitudinal accelerating gradients several orders of magnitude larger than those attainable in conventional accelerators. Especially since the first experimental evidence of monoenergetic electron spectra produced by such accelerators [2-4] interest in this field multiplied, culminating in the generation of monoenergetic electron beams with $1 \mathrm{GeV}$ energy [5]. All these experiments relied on self-injection of plasma electrons into a laser-driven plasma wave by wave breaking [6]. In this regime the intensity of the driving laser pulse is so large that the plasma wave breaks and some electrons of the background plasma are injected into the first wake of the plasma wave. The longitudinal electric field built up by the plasma wave rapidly boosts the speed of these injected electrons close to the velocity of light. They become trapped and are subsequently further accelerated to highly relativistic energies by the wave [7]. This self-injection offers the great benefit of obviating the need for the experimentally very challenging injection of an externally generated electron pulse. The inherent disadvantage of this injection technique is posed by the absence of control over the exact locations at which wave breaking-and hence injection-starts and stops and over the amount of injected charge. In order to gain some control over the injection process several different approaches have been adopted so

\footnotetext{
*karl.schmid@mpq.mpg.de

†laszlo.veisz@mpq.mpg.de
}

far: injection at a density down ramp produced electrons with a momentum of $0.76 \pm 0.02 \mathrm{MeV} / c$ [8], enhanced injection was also shown using mixtures of different gases $[9,10]$. A scheme employing a counterpropagating laser pulse $[11,12]$ was shown to improve the reproducibility of the generated electron beam.

Here we present a simple scheme that allows for precisely localized injection of electrons into a laser-driven plasma wave and subsequent acceleration yielding a monoenergetic spectrum. This scheme draws on a sharp downward (along laser propagation) plasma density transition between two adjacent regions of different densities [13]. The laser intensity and plasma density are adjusted such that the interaction is highly nonlinear but no wave breaking occurs while the laser propagates in either of the two regions. This allows for the generation of a stable but highly anharmonic plasma wave, which, in turn, provides large accelerating gradients. During the downward density transition, the plasma wavelength $\lambda_{p}$ increases abruptly from its high-density value to the low-density one. Here, the plasma wavelength is given by $\lambda_{p} \approx 2 \pi c / \omega_{p}$ and the plasma frequency by $\omega_{p}=\sqrt{n_{e} e^{2} /\left(\epsilon_{0} m_{e}\right)}$, with $e, m_{e}, n_{e}$ being electron charge, mass, and density, respectively. This sudden increase in plasma wavelength causes a rephasing of a sizable fraction of the plasma electrons into the accelerating phase of the plasma wave. We point out that this is different to the injection caused during density downramps that extend over many plasma periods [8], where a reduced plasma wave phase velocity enables effi- 
cient trapping of nonrelativistic plasma electrons. In contrast, in the case of the sharp transition, the plasma wave is fully loaded at once due to the sudden increase in plasma wavelength. Electron trapping at sharp downward density transitions has been extensively studied theoretically [1416]. Sharp in this context means that the characteristic length of the transition is on the order of the plasma wavelength [17]. Up to now experimental realizations of this scheme did not produce monoenergetic electron beams and relied on a second laser beam that induces a plasma density transition via local plasma heating [18-20].

In contrast to former schemes, in the present experimental approach the density transition is formed in the gas jet prior to ionization by the driving laser pulse. It exploits shock-front formation in a supersonic He-gas jet. The jet is generated by a pulsed de Laval nozzle with an exit diameter of $300 \mu \mathrm{m}$; the shock is generated by introducing a knife edge laterally into the gas jet. In contrast to a subsonic flow, the supersonic flow cannot adapt upstream to the obstacle and therefore needs to adapt locally in the form of an abrupt change of all flow parameters. The typical length scale of this sudden change is on the order of a few times the molecular mean free path depending on initial Mach number and shock angle [21,22]. The (asymptotic) values of the flow parameters before and after the shock can be calculated using the Euler equations. For the ratio of gas densities in front of and behind the shock, one obtains [23]

$$
\frac{n_{1}}{n_{2}}=1-\frac{2}{\kappa+1}\left(1-\frac{1}{\left(M_{1} \sin \alpha\right)^{2}}\right)
$$

where $n_{1}$ and $n_{2}$ are the gas densities before and after the shock, respectively, $\kappa$ is the specific heat ratio with a value of 5/3 for a monoatomic gas, $M_{1}$ is the initial Mach number of the gas flow, and $\alpha$ (see Fig. 1) is the angle between the gas flow and the shock front. Relation (1) exhibits a minimum for $\alpha=90^{\circ}$ corresponding to a strong perpendicular shock and a maximum value of 1 for $\alpha_{m}=$ $\arcsin \left(1 / M_{1}\right)$ for weak distortions propagating at the Mach angle. A sketch of the nozzle setup is given in Fig. 1(a). The razor blade is mounted on a small translation stage allowing to switch the shock front on and off as well as moving the shock transversally through the gas jet. Since moving the knife edge through the gas jet not only changes the shock-front position but also the angle, the density ratio changes accordingly.

The laser employed in the present experiments is the multi-TW sub-10-fs light source light wave synthesizer 20 (LWS-20) [24], an upgraded version of the system used for the experiments in [25]. During the experiments it delivered pulses with $65 \mathrm{~mJ}$ energy on target and a duration of $8 \mathrm{fs}$ full width at half maximum (FWHM). The laser pulses are focused onto the target by a f/12 off-axis parabolic mirror (OAP) to a spot diameter of $12 \mu \mathrm{m}$ (FWHM),

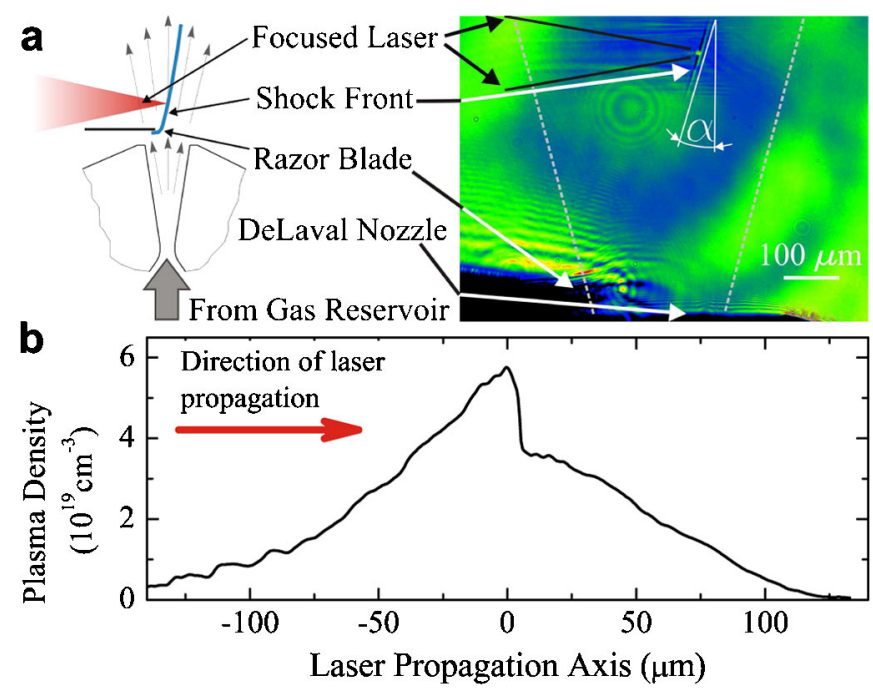

FIG. 1. Sketch and shadowgraph image of the target setup (a). A razor blade is introduced laterally into a supersonic gas jet (undistorted gas jet edges: white dashed lines) in order to generate a shock front (blue line). The shadowgraph image was taken during the experiments showing the nozzle tip, the razor blade (slightly tilted), and the plasma produced by the driving laser. Inside the plasma the tilted shock front can be discerned and a small, bright spot indicative of electron injection is visible. (b) Measured electron density along the laser propagation axis.

yielding a peak intensity of $2.5 \times 10^{18} \mathrm{~W} / \mathrm{cm}^{2}$. This value takes into account losses introduced by residual phase front distortions.

The electron energy spectrum is measured by a high resolution focusing permanent magnet spectrometer [26] suitable for analyzing electrons in the range of $2-400 \mathrm{MeV}$. Scintillating screens (Kodak Lanex) imaged to a 12 bit CCD camera are used for electron detection, allowing simultaneous measurement of energy spectrum and divergence. This detection system was absolutely calibrated at a linear accelerator source [27].

Shadowgraph images of the plasma [Fig. 1(a)] are obtained during the experiments by using a small part of the laser beam as back light. By introducing a Nomarski interferometer [28] into this probe beam, the same back light is used for interferometric measurements of the plasma density. Lineouts parallel to the shock front are taken out of the phase-shift maps produced by the measurement and subsequent Abel inversion yields the plasma density distribution. It was confirmed that the tilt by the angle $\alpha$ of the lineouts with respect to the plasma channel does not significantly change the results. A density lineout along the laser axis is shown in Fig. 1(b).

Figures 1(a) and 1(b) yield $\alpha=16 \pm 0.5^{\circ}$ and $n_{1} / n_{2} \simeq$ 1.6. Substitution into formula (1) gives a Mach number $\mathrm{M}=5.1 \pm 0.2$ which is in good agreement with $\mathrm{M}=5.3$ obtained from computational fluid dynamics simulations. 
TABLE I. Comparison self-injection and shock-front injection.

\begin{tabular}{lcc}
\hline \hline Parameter & Self-injection & Density transition \\
\hline Energy (MeV) & $26.0_{-6.6}^{+8.2}$ & $23.3_{-3.0}^{+3.3}$ \\
Energy spread (\%) & $12_{-10}^{+9}$ & $9_{-8}^{+6}$ \\
Divergence (mrad) & $10.9_{-3.7}^{+3.5}$ & $8.9_{-3.3}^{+3.1}$ \\
Charge (pC) & $3.7_{-3.1}^{+2.9}$ & $3.3_{-2.2}^{+2.0}$ \\
\hline \hline
\end{tabular}

For the resultant effective Mach number $\mathrm{M}_{1} \sin (\alpha)$ of 1.4, the width of the hydrodynamic shock is expected to be roughly 1 order of magnitude larger than the molecular mean free path [22] which for He at a neutral gas density of $1.9 \times 10^{19} \mathrm{~cm}^{-3}$ is approximately $0.6 \mu \mathrm{m}$. From Fig. 1 we evaluate the width of the density transition as $\approx 5 \mu \mathrm{m}$, in agreement with this expectation.

After fine-tuning the plasma density and focal spot position, quasimonoenergetic electron energy spectra are obtained. As in [25], the spectra are virtually backgroundfree. Depending on the day of operation, the occurrence probability of a quasimonoenergetic electron spectrum varies between $50 \%$ and $95 \%$. In order to quantify the improvement obtained with this new injection scheme, Table I shows a comparison of electron beam parameters produced by self-injection and by shock-front injection. Errors are root-mean-square (RMS) deviations from the average value. To account for asymmetric distributions, the RMS value was independently calculated for the occurrences with higher-than-average and lower-than-average value. In Fig. 2 and Table I, energy spread and divergence refer to FWHM.
It can be seen that for density-transition injection, all shot-to-shot fluctuations as well as the energy spread and divergence are smaller than in the self-injection case. In both cases, approximately $36 \%$ of all the shots fall within the RMS error intervals with respect to all four parameters simultaneously. Since these error intervals are much smaller for the density-transition case, this again shows a clear advantage of injection at sharp density transitions.

Representative electron spectra out of the $10 \%$ fraction with lowest energy spread are displayed in Fig. 2. It is evident that the shock-front injection scheme yields a much cleaner and more reproducible beam than obtainable with self-injection. The accelerator is routinely operated at the laser repetition rate of $10 \mathrm{~Hz}$; therefore, the electron accelerator currently produces approximately one high quality shot per second on a daily basis and is, thus, ready for first applications. To support the claim that electron injection is indeed happening exclusively at the location of the shock front, we note first that at the crossing point between the laser beam and the shock front a bright spot of broadband light emission occurs in the side view images [see Fig. 1(a)] resembling the wave-breaking radiation reported in [29]. It was confirmed that the brightness of the spot is correlated to the accelerated charge. Further support is provided by the fact that the electron energy scales linearly with the position of the shock front and, hence, with the acceleration length. In this way the electron energy could be varied between 15 and $23 \mathrm{MeV}$; the accelerating gradient was determined as $\sim 190 \mathrm{GV} / \mathrm{m}$. This result is presented in Fig. 3.

Numerical simulations with the three-dimensional particle-in-cell (3D-PIC) code ILLUMINATION [30] pro-

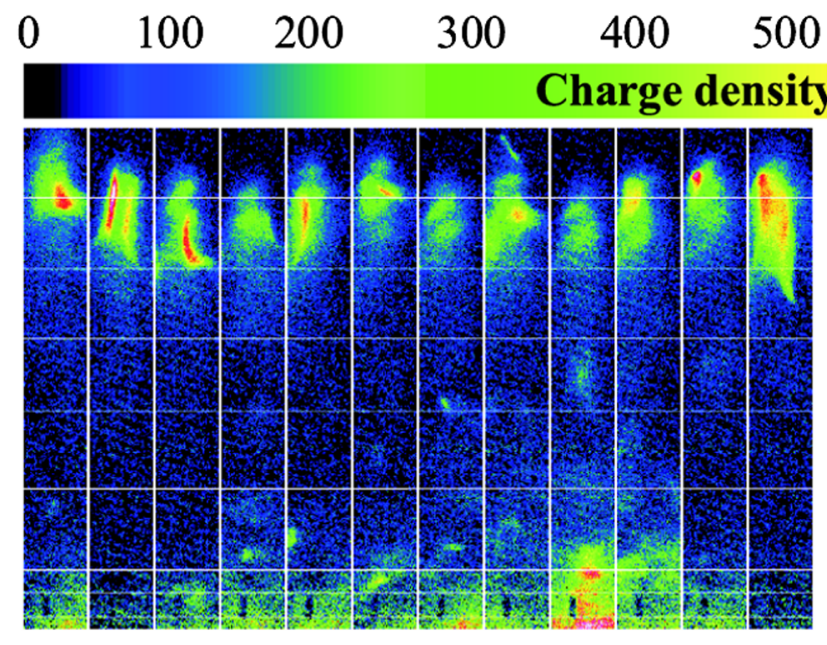

(a) Self injection

\section{$\begin{array}{llllll}600 & 700 & 800 & 900 & 1000 & 1100\end{array}$}
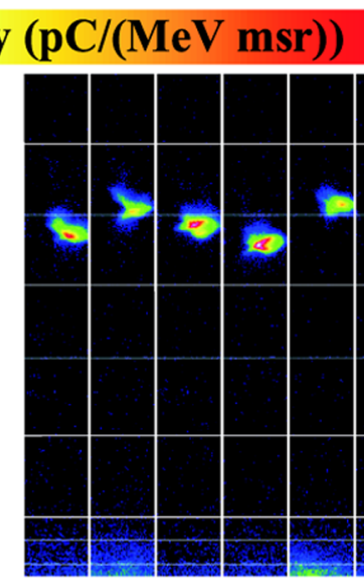

(b) Injection at density transition

FIG. 2. A few shots representative for those $10 \%$ of all the shots with lowest energy spread for self-injection (a) and injection at a density transition (b). The horizontal axis in each image corresponds to the transversal electron beam size; the vertical axis shows electron energy. For self-injection the parameters of this fraction are $29.7 \pm 1.2 \mathrm{MeV}$ energy, $8 \pm 5 \%$ energy spread, $10.0 \pm 2.3 \mathrm{mrad}$ divergence, and $3.1 \pm 1.0 \mathrm{pC}$ charge. For injection at the density transition, these values are $24.3 \pm 0.9 \mathrm{MeV}, 4 \pm 0.5 \%, 7.3 \pm$ $0.5 \mathrm{mrad}$, and $1.8 \pm 0.5 \mathrm{pC}$, respectively. 


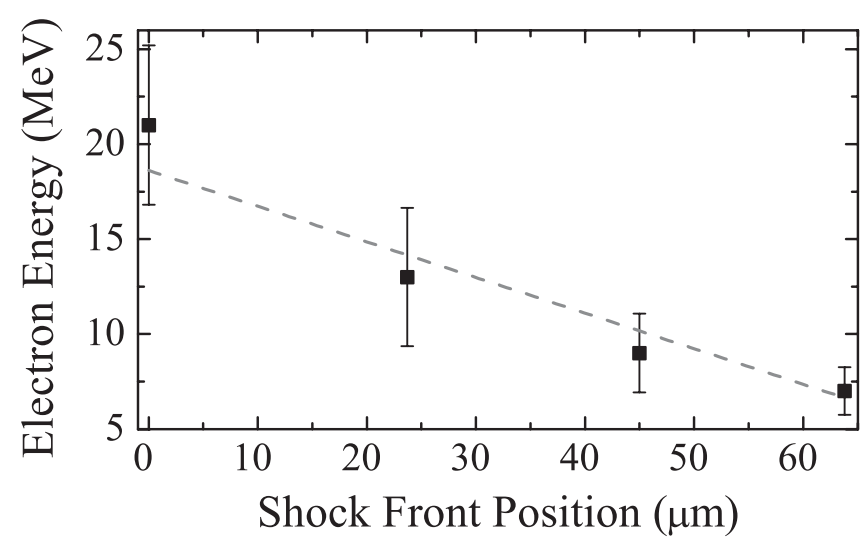

FIG. 3. Variation of electron energy with the position of the density transition. The grey dashed line shows a linear fit yielding an acceleration gradient $\simeq 190 \mathrm{GV} / \mathrm{m}$.

vided further details of the injection process. The laser pulse and plasma parameters used in the simulation match those of the experiment. Figure 4 shows, from top to bottom, the physical state of the laser-driven plasma wave shortly before, immediately after and $\sim 85$ fs after the laser pulse crossed the downward density transition. The plasma wave is strongly driven by the laser pulse, but does not break until the laser pulse crosses the density transition. At the transition the wave breaks and a compact electron bunch with $60 \mathrm{pC}$ charge is injected into the first wave bucket after the laser pulse, leading to a complete destruction of the following plasma wave buckets. This is to say, the wave fully breaks down at this point, leaving only the accelerating bubble structure. The accelerating field at the position of the electron bunch is $250 \mathrm{GV} / \mathrm{m}$, in reasonable agreement with the experimental value. The PIC simulations confirm that electrons are exclusively injected at the density transition and show the injection mechanism to be quite robust against changes of the upper and lower density levels, a fact which was also confirmed experimentally.

In conclusion, we present a simple, reliable, and robust method of injecting background electrons into a laserdriven plasma wake. It relies on injection at a sharp downward density transition originating from a shock front which is generated by a razor blade introduced laterally into a supersonic gas jet. The resulting electron beam is typically monoenergetic and has a significantly improved stability and reduced energy spread. Additionally, the electron energy can be tuned in a wide range. It is important to note that the present setup is easily adapted to different laser parameters and-once applied to multi-Joule laser pulses - could pave the way towards a reliable tabletop electron accelerator in the multi-100 MeV range. By generating a supersonic shock front inside a guiding structure like a capillary [5], even the GeV energy range comes into reach.

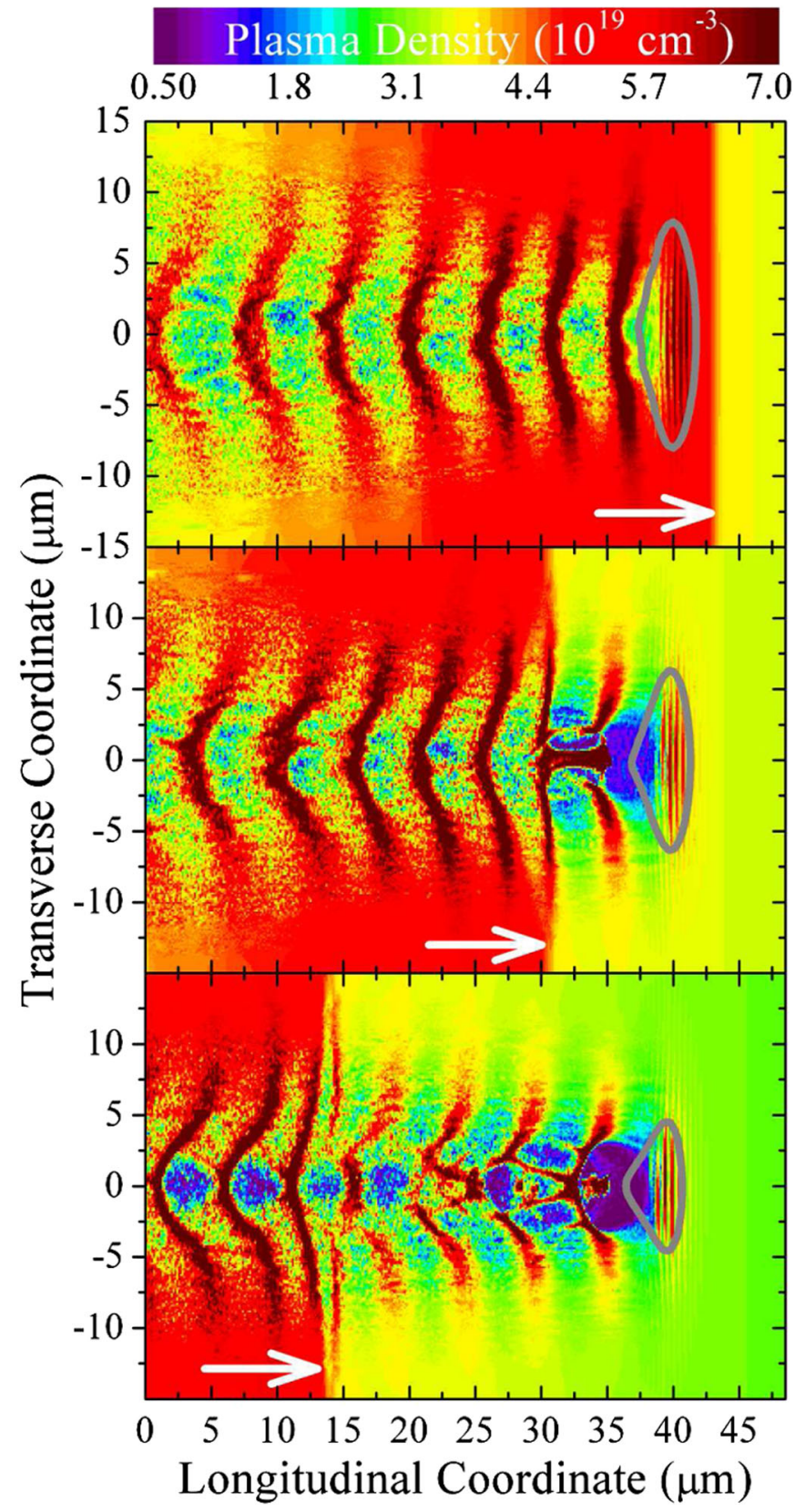

FIG. 4. State of the accelerator as obtained from PIC simulations shortly before (upper panel), immediately after (middle panel), and $\sim 85$ fs after (lower panel) the laser pulse has crossed the density transition. White arrows indicate the position of the density transition; grey lines show the contour where $13.5 \%$ of maximum laser intensity is reached. The normalized instantaneous laser intensity is shown in a rainbow color map. The upper panel shows a strongly driven plasma wave that does not break. Strong injection triggered by the shock front is evident in the middle panel. The strong electric field of the injected electron bunch destroys the remaining plasma wave thus impeding injection in more than one wave-trough. This leads to a fully developed bubble structure (lower panel) that accelerates the injected electrons to several $10 \mathrm{MeV}$ energy. Since the bubble is fully loaded with electrons, no further injection occurs. 


\section{ACKNOWLEDGMENTS}

This work is supported by DFG-Project Transregio TR18, by the Association EURATOM-Max-PlanckInstitut fuer Plasmaphysik, by The Munich Centre for Advanced Photonics (MAP) and by the Laserlab-Europe/ Labtech FP7 Contract No. 228334. C. M. S. Sears acknowledges the support of the Alexander von Humbold Foundation. J. M. Mikhailova acknowledges support by Alexander-von-Humboldt Foundation and RFBR, Grants No. 08-02-01245-a and No. 08-02-01137-a. D. Herrmann is grateful to Studienstiftung des deutschen Volkes.

[1] T. Tajima et al., Phys. Rev. Lett. 43, 267 (1979).

[2] C. Geddes et al., Nature (London) 431, 538 (2004).

[3] S. Mangles et al., Nature (London) 431, 535 (2004).

[4] J. Faure et al., Nature (London) 431, 541 (2004).

[5] W. P. Leemans et al., Nature Phys. 2, 696 (2006).

[6] S. V. Bulanov et al., Phys. Rev. Lett. 78, 4205 (1997).

[7] A. Pukhov et al., Appl. Phys. B 74, 355 (2002).

[8] C. Geddes et al., Phys. Rev. Lett. 100, 215004 (2008).

[9] A. Pak et al., Phys. Rev. Lett. 104, 025003 (2010).
[10] C. McGuffey et al., Phys. Rev. Lett. 104, 025004 (2010).

[11] J. Faure et al., Nature (London) 444, 737 (2006).

[12] C. Rechatin et al., Phys. Rev. Lett. 102, 164801 (2009).

[13] S. Bulanov et al., Phys. Rev. E 58, R5257 (1998).

[14] H. Suk et al., Phys. Rev. Lett. 86, 1011 (2001).

[15] P. Tomassini et al., Phys. Rev. ST Accel. Beams 6, 121301 (2003).

[16] H. Suk et al., J. Opt. Soc. Am. B 21, 1391 (2004).

[17] A. V. Brantov et al., Phys. Plasmas 15, 073111 (2008).

[18] J. Kim et al., Phys. Rev. E 69, 026409 (2004).

[19] T.-Y. Chien et al., Phys. Rev. Lett. 94, 115003 (2005).

[20] J. Kim et al., J. Korean Phys. Soc. 51, 397 (2007).

[21] D. Haenel, Molekulare Gasdynamik (Springer-Verlag, Berlin, 2004), 1st ed.

[22] H. M. Mott-Smith, Phys. Rev. 82, 885 (1951).

[23] R.D. Zucker and O. Biblarz, Fundamentals of Gas Dynamics (Wiley, New York, 2002).

[24] D. Herrmann et al., Opt. Lett. 34, 2459 (2009).

[25] K. Schmid et al., Phys. Rev. Lett. 102, 124801 (2009).

[26] C. M. S. Sears et al. (to be published).

[27] A. Buck et al., Rev. Sci. Instrum. 81, 033301 (2010).

[28] R. Benattar et al., Rev. Sci. Instrum. 50, 1583 (1979).

[29] A. Thomas et al., Phys. Rev. Lett. 98, 054802 (2007).

[30] M. Geissler et al., New J. Phys. 8, 186 (2006). 